\title{
Very High Energy Observations of BL Lacs
}

\author{
John Quinn, Peter Cogan, Michael K. Daniel, David J. Fegan, \\ Stephen Gammell and Andrew McCann
}

School of Physics, University College Dublin, Belfield, Dublin 4, Ireland email: john.quinn@ucd.ie

\begin{abstract}
The status of Very High Energy (VHE, E > $50 \mathrm{GeV}$ ) gamma-ray observations of BL Lacertae objects is presented. The catalogue of well-established BL Lacertae objects detected at VHE energies contains seven members, and there have been recent reports of the detection of another four. All are nearby, X-ray bright sources. The temporal, spectral and broadband multiwavelength properties of the sources are reviewed and possible implications for the gamma-ray production mechanism discussed. The most recent detections provide more stringent constraints on the cosmic extragalactic background light level and imply that the Universe is more transparent to VHE gamma radiation than previously thought.
\end{abstract}

Keywords. BL Lacertae objects: individual, gamma rays: observations.

\section{Introduction}

The maturing of the Imaging Atmospheric Cherenkov Technique (IACT) has opened a new astronomical window on the cosmos with observations in the $50 \mathrm{GeV}-50 \mathrm{TeV}$ now routinely being made with ground-based instruments. Large optical reflectors are used to image the Cherenkov light emitted by extensive air showers in the atmosphere and off-line image analysis techniques distinguish between gamma-ray and charged cosmicray events. The technique has been used to detect in excess of 30 sources of VHE gamma rays, including $11 \mathrm{BL}$ Lacs. For an introduction to the field of ground-based gamma-ray astronomy see Catanese and Weekes (1999) and Cogan et al. (2005).

BL Lac objects are a subset of the blazar class of Active Galactic Nuclei, and are characterised by a featureless optical continuum while exhibiting rapid variability. The blazar class, which also contains Flat-Spectrum Radio Quasars (FSRQs), are believed to be powerful radio galaxies which have their jets oriented towards the Earth. Blazars are very prominent in the gamma-ray sky; the EGRET detector on board the Compton Gamma-Ray Observatory detected 50 such objects above $100 \mathrm{MeV}$, (almost $40 \%$ of the entire EGRET third catalogue), 14 of which are BL Lacs with the rest being FSRQs. Thus, the EGRET sources were strong candidates to be VHE emitters and were amongst the first objects to be investigated with IACT instruments.

\section{VHE Observations}

\subsection{The VHE Catalogue}

There are seven well-established and well-studied BL Lacs in the VHE band. These are listed in Table 1. In addition, at the recent International Cosmic-Ray Conference in India an additional four detections (Table 2) were announced. All belong to the sub classification called HBL (High Frequency BL Lacs) which refers to the x-ray emission from these objects showing a peak at $\mathrm{keV}$ energies. The first objects detected were the closest (Markarian 421 and Markarian 501) but as more extensive surveys were carried 
Table 1. Established VHE BL Lacs

\begin{tabular}{lccccc}
\hline Catalogue Name & Source & Date & Group & Type & Redshift \\
\hline TeV 1104+3813 & Markarian 421 & 1992 & Whipple & HBL & 0.031 \\
TeV 1429+4240 & H1426+428 & 2002 & Whipple & HBL & 0.129 \\
TeV 1654+3946 & Markarian 501 & 1995 & Whipple & HBL & 0.033 \\
TeV 2000+6509 & 1ES1959+650 & 1999 & Telescope Array & HBL & 0.048 \\
TeV 2005-489 & PKS 2005-498 & 2005 & HESS & HBL & 0.071 \\
TeV 2159-3014 & PKS 2155-304 & 1999 & Durham & HBL & 0.116 \\
TeV 2347-5142 & 1ES2344+514 & 1997 & Whipple & HBL & 0.044 \\
\hline
\end{tabular}

Table 2. Recently announced VHE BL Lacs

\begin{tabular}{lcccc}
\hline Source & Date & Group & Type & Redshift \\
\hline PKS 2005-489 & 2005 & HESS & HBL & 0.071 \\
H 235-309 & 2005 & HESS & HBL & 0.165 \\
1ES 1101-232 & 2005 & HESS & HBL & 0.186 \\
1ES 1218+304 & 2005 & Magic & HBL & 0.182 \\
\hline
\end{tabular}

out and increasingly sensitive instruments came on-line the horizon was extended. The most distant BL Lac detected at VHE energies is $1 \mathrm{ES} 1101-232$ at $\mathrm{z}=0.186$.

\subsection{Flux Variability}

As at other wavelengths, the BL Lac emission at VHE energies is characterised by variability. The time scales for significant flux change range from years to less than one hour in some cases. Figure 1 (top) illustrates this for two sources; Markarian 421 and Markarian 501. In 1996 Markarian 421 was observed to undergo a series of flares which were remarkable for their amplitudes as well as their rapidity; on May 15 Markarian 421 went from being virtually undetectable to become the brightest known object at VHE energies in the space of less than an hour, and then rapidly faded to a low flux state. In 1997 Markarian 501, after a few years of relative inactivity, underwent a series of rapid flaring with dramatic month- and day-scale flaring with evidence for variability on a time-scale of hours. Monitoring of all the other established VHE BL Lacs also revealed significant variability with little evidence of any steady base-line emission.

\subsection{Spectral Properties}

The VHE energy spectra can all be fit either by power laws or power laws with exponential cutoffs:

$$
\frac{\mathrm{d} N}{\mathrm{~d} E} \propto E^{-\Gamma} e^{-E / E_{0}}
$$

Measured spectral indices of the established BL Lacs range from $\Gamma=1.9$ to $\Gamma=3.5$ with a tendency for a harder spectrum with increasing distance (Schroedter, 2005). The cut-off energy for Markarian 421 has been measured to be $\approx 4.3 \mathrm{TeV}$ (Krennrich et al., 2002), while for Markarian 501 has been found to be $\approx 6 \mathrm{TeV}$ (Aharonian et al., 1999). In addition, Markarian 421 was monitored at a variety of different flux levels and spectral analysis revealed that there was a correlation between spectral index and flux level, with harder indices associated with higher flux levels (Krennrich et al., 2002). Also, it was found that there was no change in the cut-off energy with flux.

Broadband multiwavelength observations have been carried out on several of the established VHE BL Lacs. The general trend appears to be that there is some correlation between the x-ray and gamma-ray emission. However, some 'orphan' flares have been 

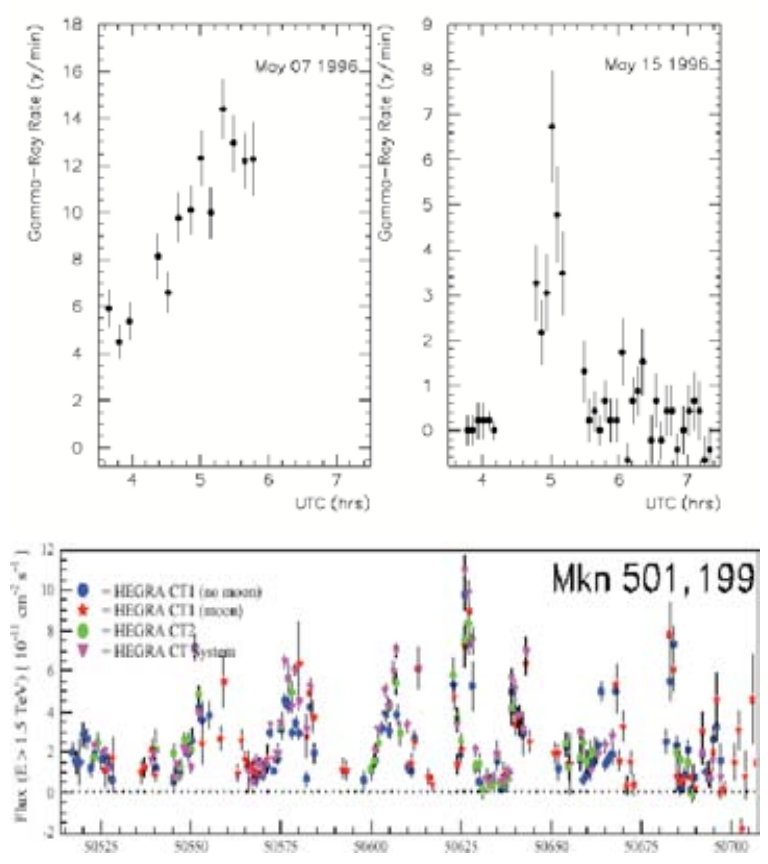

Figure 1. Top: Two remarkable flaring episodes from Markarian 421 in 1996 (Gaidos et al., 1996). Bottom: daily fluxes from Markarian 501 in 1997 (Kranich et al., 2001).

observed (e.g. Krawczynski et al., 2004), where a flare is seen in either the X-ray or gamma-ray band but not both simultaneously. In general the broadband spectral energy distribution of BL Lacs consists of a double-peaked structure, as illustrated in Figure 2.

\section{Implications of VHE BL Lac Detections}

\subsection{Gamma-ray production mechanism}

There are two main classes of models for gamma-ray production in BL Lac jets, distinguished by the dominant particle species in the jet. In one class it is electrons that are believed to produce the gamma radiation. It is generally accepted that the first peak is due to beamed incoherent synchrotron radiation from electrons. These electrons can then inverse-Compton scatter photons to gamma-ray energies. In the Synchrotron SelfCompton model the electrons scatter the synchrotron photons that they produced while in the External Inverse-Compton model the seed photons originate from outside the jet. Conversely, in the hadronic family of models protons are responsible for the gammaray emission, either through direct synchrotron radiation or through the decay of pions which occur when the protons collide with target material. As yet, no class of models have been ruled out but the multi-wavelength observations indicate that the same particles which produce the synchrotron photons may also produce the gamma-ray photons, hence favouring electron models.

\subsection{Cosmic Extragalactic Background Light}

VHE gamma rays interact with low energy photons to produce electron-positron pairs. For a $1 \mathrm{TeV}$ gamma ray the cross section is a maximum when the wavelength of the soft photon is $1.33 \mu \mathrm{m}$. Thus VHE photons will be attenuated on their journey from extragalactic sources and the spectrum of VHE photons modified. Whilst this presents 


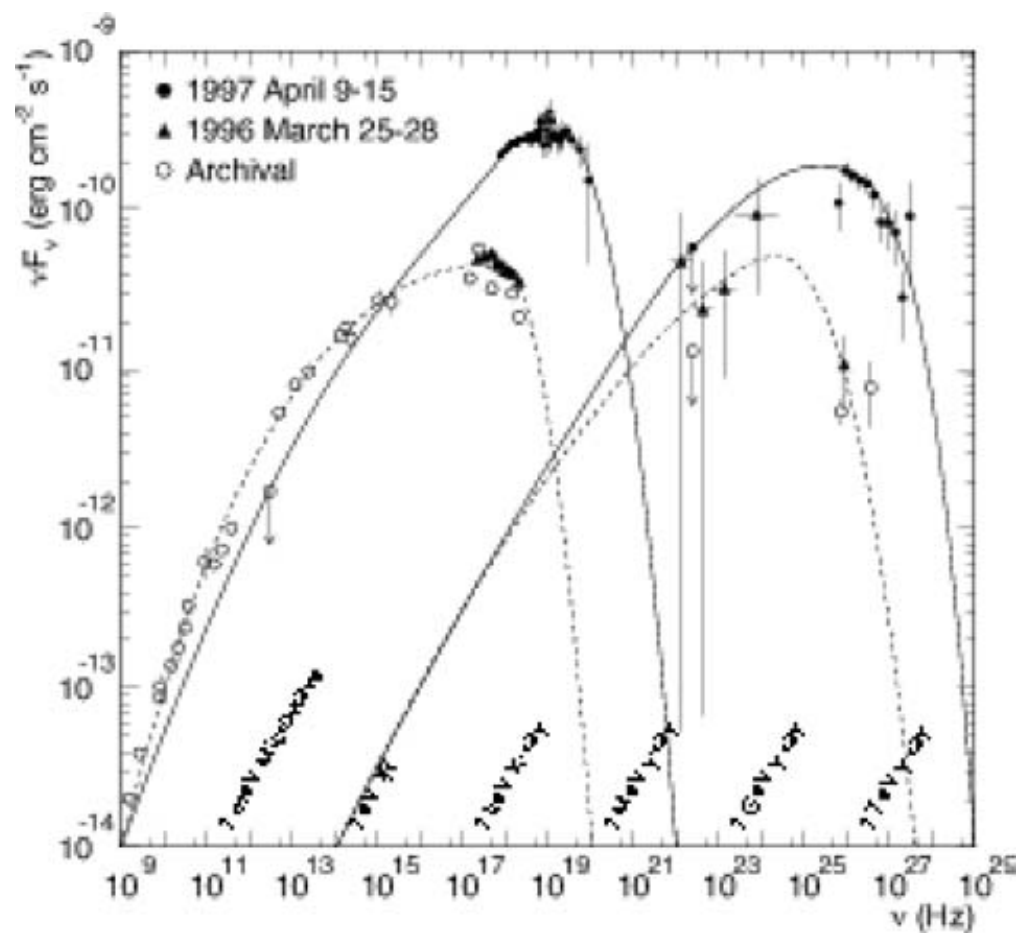

Figure 2. Broadband Spectral Energy Distribution of Markarian 501, from Catanese and Weekes (1999).

a difficulty for extragalactic VHE astronomy, as distant sources are difficult to detect, it also provides a mechanism to probe the cosmic EBL density, of which direct measurement is extremely difficult. The most recent results by HESS (Aharonian et al., 2005) indicate that the EBL must be quite low, at the limit of that expected from galaxy source counts, and that the Universe is more transparent to VHE gamma rays than previously thought.

\section{Conclusions and future prospects}

The field of BL Lac astrophysics has been reinvigorated by the results from VHE instruments. There are now claimed detections of 11 objects, with 7 having been detected by multiple experiments. Third-generation IACT experiments, currently either online or coming coming online, are expected to dramatically expand our catalogue of gamma-ray emitting BL Lacs and allow more detailed studies of individual objects.

\section{References}

Aharonian, F., et al., 1999, A\& A, 349, 11

Aharonian, F., et al., 2005, submitted to Nature, astro-ph/0508073

Catanese, M. and Weekes, T.C., 1999, PASP, 111, 1193

Cogan, P., et al., these proceedings

Gaidos, J., et al., 1996, Nature, 383, 319

Kranich, D., et al., 2001, Proc. 27th ICRC (Hamburg)

Krawczynski, H. et al., 2004, ApJ, 601, 151

Krennrich, F., et al., 2002, ApJ, 575, L9

Schroedter, M., 2005, ApJ., 628, 617S 


\section{Discussion}

MirABEL: Can you explain the blazer phenomenology with bulk Lorentz factors of a few or you need longer than AGN?

QUINN: In general higher Lorentz factors $(\sim 50-100)$ are needed to fit one spectrum and variability observed $\sim \mathrm{TeV}$ blazers.

Courvoisier: Do you take the IR radiation of the stars of the host galaxies when deducing the intergalactic IR background?

QUINN: This could explain why certain types of AGN are not seen at TeV easier. However, if absorption is occurring at the source then our estimates of the IR background level are underestimated.

Meurs: You showed extensive TeV lightcurve data of Mark 501. Have there been any attempts to perform a power spectrum analysis of these data to search for any periodicities?

QUinN: Some individuals analysed the data and claimed that there was evidence for a 23-day periodicity. Given that Cherenkov telescopes cannot operate in moonlight a 20something day periodicity may be expected. The HEGRA collaboration themselves do not claim that there is any periodicity in the data.

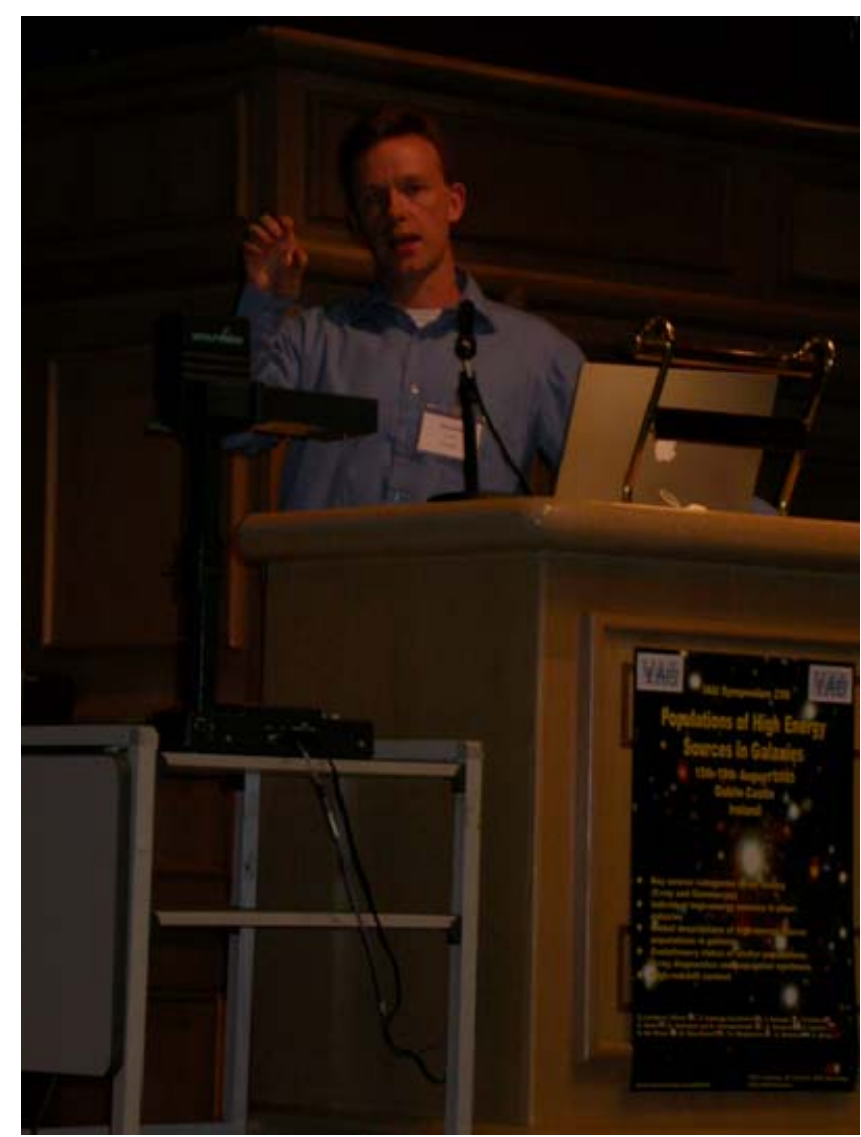

John Quinn indicates a black hole size. 